Check for updates

Cite this: RSC Adv., 2021, 11, 18299

\title{
Novel hydrophobic catalysts to promote hydration at the water-oil interface
}

\author{
Shuai Liu, Dahai Sun and Hui Tian (D)* \\ The limitation of the cyclohexene hydration reaction is that it is a three-phase immiscible reaction. We have \\ described a strategy to overcome this interfacial mass transfer limitation by grafting an organosilane \\ surfactant ((octyl)-trimethoxysilane (OTS)) onto the HZSM-5 zeolite surface. The characterization of the \\ OTS-HZSM-5 zeolite was performed by FTIR, CA, BET, TPD, pyridine-IR, XPS, TGA and XRD techniques. \\ The functionalization of the HZSM-5 zeolite could increase hydrophobicity without significantly reducing \\ the density of acid sites. As a result, the OTS-HZSM-5 zeolite had high catalytic activity $(20.87 \%$ \\ conversion) compared with HZSM-5 (4.15\% conversion) at $130{ }^{\circ} \mathrm{C}$ after $4 \mathrm{~h}$. The high catalytic activity \\ makes it a promising candidate for other acid-catalyzed two-phase reactions.
}

Received 13th February 2021

Accepted 29th April 2021

DOI: $10.1039 / \mathrm{d} 1 \mathrm{ra01188j}$

rsc.li/rsc-advances

A method without reducing the acid site density is the sily-

\section{Introduction}

Cyclohexanol is an important intermediate material for the production of adipic acid and caprolactam. Adipic acid is used as an important raw material for polymerization to produce nylon 66 and synthetic polyurethane, and caprolactam is used in the production of engineering plastics. ${ }^{1-6}$ Cyclohexanol is often used as a solvent for paints and varnishes in coatings, and as a matting agent in textiles. ${ }^{7,8}$ Therefore, cyclohexanol is an important chemical raw material.

Cyclohexene hydration is a heterogeneous immiscible reaction, in which the solid catalyst is at the bottom of the water phase, which cannot contact cyclohexene. ${ }^{9-13}$ In fact, numerous scholars have done research on the modification of ZSM-5. Spod et $a .^{14}$ used HZSM-5 as a catalyst for cyclohexene hydration, in which the cyclohexene conversion could reach up to $13 \%$. Nur et al. ${ }^{15}$ carried out the silylation of HZSM-5 and NaY with sulfanilic acid to prepare 1,2-epoxyoctane. They found that the active sites were mainly located on the inner surface of the zeolite. Therefore, they implemented the concept of the amphiphilic catalyst by partially covering it to improve conversion. A study by Shaodan Xu et al. ${ }^{16}$ showed that the hydrophobic zeolites were prepared by hexadecyltrimethoxysilane (C16). The contact angle of ZSM-5 was improved to $134^{\circ}$ by functionalization. Zapata et al. ${ }^{17}$ recently obtained the hydrophobic HY zeolite by functionalization with organosilanes, which is more stable in hot water than the untreated zeolite. The hydrophilicity/hydrophobicity of the zeolite received increased attention due to its significant influence on the physical and chemical properties and application of zeolites. ${ }^{18-23}$

College of Chemistry and Chemical Engineering, Yantai University, Yantai 264005, China.E-mail: tianhui@ytu.edu.cn; Tel: +8618754591289 lation of the external surface using organosilanes, which could increase hydrophobicity. ${ }^{24} \mathrm{~A}$ remarkable characteristic of these material is their high affinity towards liquid-liquid interfaces, which stabilizes the emulsions of small droplet size. Hydrophobic materials could form oil-in-water and water-in-oil in a water-oil environment, which greatly increase the liquidsolid-liquid interface area and facilitate the separation of molecules from the reaction system. ${ }^{25}$

In this study, the silylation of the zeolite is shown to be significantly more active in cyclohexene hydration. To functionalize the silanol groups, the OTS-HZSM-5 zeolite was prepared by (octyl)-trimethoxysilane (OTS). Compared to other silylation reagents, the advantage of OTS is that it has three methoxy groups. We proposed the "triangle $\mathrm{Si}-\mathrm{O}-\mathrm{Si}$ " bond hypothesis as it could form a "triangular $\mathrm{Si}-\mathrm{O}-\mathrm{Si}$ " bond with the zeolite surface, which the three methoxy groups could combine with silanol groups to form a three-dimensional triangle. Simultaneously, the adjacent OTS was connected by "Si-O-Si" bonds to ensure the steady adsorption of the $\mathrm{C} 8$ hydrophobic chain. Since the catalyst was in the middle of the phase interface, the hydration system could form a Pickering emulsion by heating. The Pickering emulsion enhanced the interfacial mass transfer by increasing the contact surface between the two immiscible reactants. ${ }^{26}$ The OTS-HZSM-5 zeolite stabilized water/oil emulsion to solve the limitation in the hydration process of cyclohexene.

\section{Materials and methods}

\subsection{Functionalization of zeolites with organosilanes}

The HZSM-5 zeolite was obtained from Nankai University, China. We chose this HZSM-5 zeolite with $25 \mathrm{Si} / \mathrm{Al}$ ratio because it has a high acid site density to better promote cyclohexene 
hydration. (Octyl)-trimethoxysilane (OTS) was obtained from MACKLIN. Methylbenzene, ethyl alcohol and carbon tetrachloride were provided by SINOPHARM.

For the functionalization of the silanol groups, $1.65 \mathrm{~g}$ water was dispersed in $100 \mathrm{~mL}$ methylbenzene by sonication using a Horn sonicator (Fisher Scientific $600 \mathrm{~W}, 20 \mathrm{kHz}$ ) at $35 \%$ amplitude. It was then stirred by a magnetic stirrer (CJJ78-1) for 20 min. $5 \mathrm{~g}$ of the untreated HZSM-5 zeolites were added into the mixture and stirred for $30 \mathrm{~min}$. Further, a $1.65 \mathrm{~g}$ solution of OTS was added in the suspension. The mixed liquor was stirred for $1 \mathrm{~h}$ at room temperature. Then, the zeolite was collected through filtration using a nylon filter $(0.22 \mu \mathrm{m}$ pore size $)$, followed by washing with ethyl alcohol and carbon tetrachloride. The silylation of the zeolite was dried at $120{ }^{\circ} \mathrm{C}$. This zeolite was named OTS(25\%)-HZSM-5. The hydrophobic functionalization of the HZSM-5 zeolite is shown in Fig. 1.

Different loadings of (octyl)-trimethoxysilane (OTS) were prepared, but the amount of OTS and water were difference. For OTS(20\%)-HZSM-5, $1.25 \mathrm{~g}$ solution of OTS and $1.25 \mathrm{~g}$ of water were chosen; for OTS(25\%)-HZSM-5, $1.65 \mathrm{~g}$ solution of OTS and $1.65 \mathrm{~g}$ of water were chosen; for OTS(30\%)-HZSM-5, $2.15 \mathrm{~g}$ solution of OTS and $2.15 \mathrm{~g}$ of water were chosen; for OTS(35\%)HZSM-5, $2.69 \mathrm{~g}$ solution of OTS and $2.69 \mathrm{~g}$ of water were chosen; for OTS(40\%)-HZSM-5, $3.35 \mathrm{~g}$ solution of OTS and $3.35 \mathrm{~g}$ of water were chosen.

$$
\begin{aligned}
\text { Silylation }(\%)= & \frac{\text { organosilane quality }}{\text { organosilane quality }+ \text { catalyst quality }} \\
& \times 100 \%
\end{aligned}
$$

\subsection{Study of cyclohexene hydration}

The hydration of cyclohexene was performed in a $100 \mathrm{~mL}$ stainless-steel reactor on oil bath, to which water, catalysts and cyclohexene were sequentially added. The catalyst evaluations were carried out under different conditions. The reactants and products were analyzed via gas chromatography (7890B GC;
Agilent Technologies, Santa Clara, CA, USA) and equipped with a mass spectrometer and flame ionization detector for product identification and quantification, respectively. The cyclohexene conversion was calculated as follows:

$$
\text { Xcyclohexene }(\%)=\frac{\text { mass of cyclohexene reacted }}{\text { initial mass of cyclohexene }} \times 100 \%
$$

\section{Results and discussion}

We first confirmed the successful grafting of OTS onto the HZSM-5 zeolite and characterized the changes in the surface properties. These analyses were performed via Fouriertransform infrared spectroscopy (FTIR), contact angle (CA), $\mathrm{N}_{2}$-adsorption/desorption (BET), temperature-programmed desorption (TPD), pyridine-IR, X-ray photoelectron spectroscopy (XPS), thermal gravimetric analysis (TGA) and X-ray diffraction (XRD) techniques. Then, we evaluated the effects of OTS grafting onto the HZSM-5 zeolite in the cyclohexene hydration reaction under various conditions. According to the catalyst evaluation result, HZSM-5 and OTS(25\%)-HZSM-5 were chosen for characterization measurements.

\subsection{Characterization of the organosilane-grafted HZSM-5 zeolites}

3.1.1 Fourier-transform infrared spectroscopy (FTIR) analysis. The FTIR spectra confirmed the successful grafting of OTS onto the HZSM-5 zeolite. The skeletal FTIR spectra of the OTSHZSM-5 zeolites presented an asymmetric stretching of the $\mathrm{C}-\mathrm{H}$ bridges at 2800 and $3000 \mathrm{~cm}^{-1}$ (Fig. 2), which is attributed to the stretching vibrations of OTS. ${ }^{27,28}$ The breaking of the hydrogen bonds on the zeolite surface could form "Si-O-Si" bonds, which led to the $\mathrm{C} 8$ hydrophobic chain get adsorbed on the zeolite surface. ${ }^{29}$ Therefore, the new peaks indicated that a densely hydrophobic layer was formed on the zeolite surface. From Fig. 2, the Bronsted acid sites can be seen at $3610 \mathrm{~cm}^{-1}$,

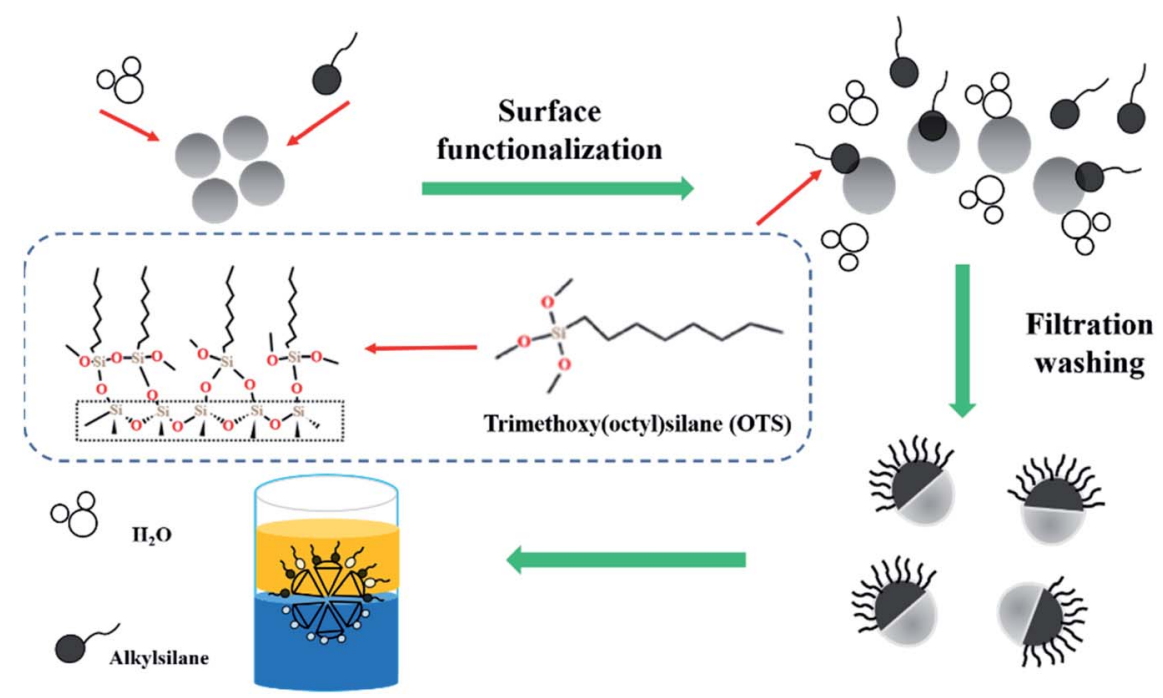

Fig. 1 Hydrophobic functionalization of the HZSM-5 zeolite. 


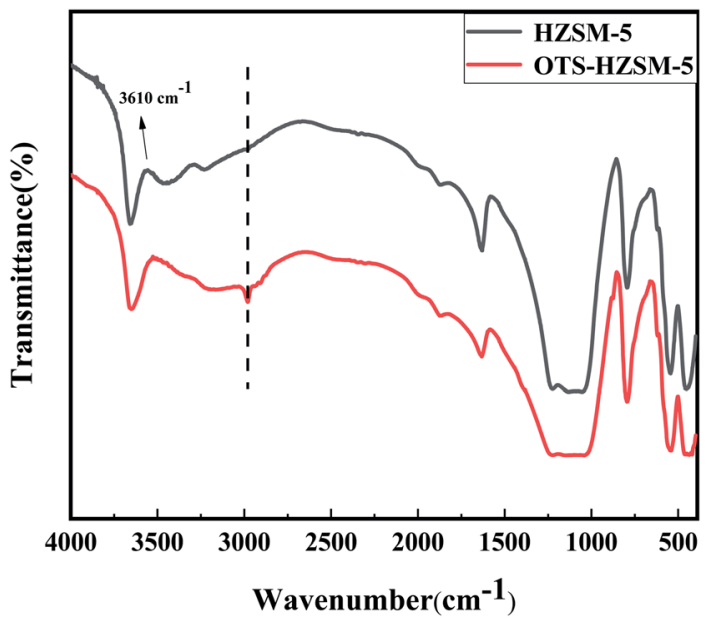

Fig. 2 FT-IR spectra of HZSM-5 zeolite and OTS-HZSM-5 zeolite (Si/ $\mathrm{Al}=25)$.

and this result is in agreement with information obtained in the study by Qin Zhengxing et al. ${ }^{30}$ Compared with the spectra of HZSM-5, the absorption intensity of hydroxyl groups decreased in OTS-HZSM-5, which indicated that surface wettability may have changed from hydrophilic to hydrophobic.

3.1.2 Contact angle analysis. The contact angle (CA) was measured to determine the degree of hydrophobicity of the OTS-HZSM-5 zeolite. As shown in Fig. 3, the samples with the untreated HZSM-5 zeolite showed good hydrophilicity with a CA of $16.28^{\circ}$. The C8 hydrophobic chain could improve the hydrophobicity of the zeolite and lead the CA of OTS-HZSM-5 zeolites exceeded $20^{\circ}$. The CA results were different from the report, which the CA of the ZSM-5 zeolite was as high as $\sim 150^{\circ}$
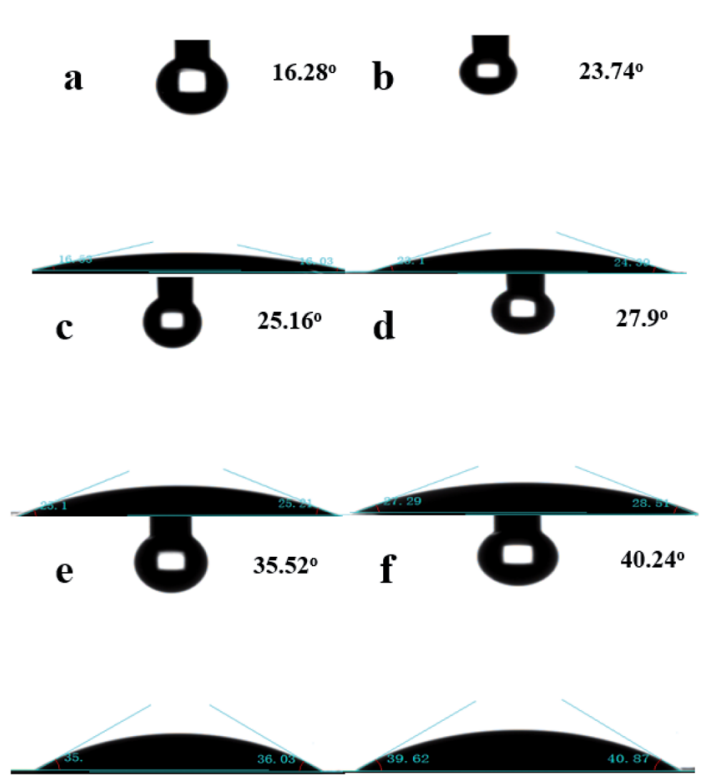

Fig. 3 Contact angle variation on HZSM-5 zeolites at different amounts of OTS: (a) untreated HZSM-5, (b) OTS(20\%)-HZSM-5, (c) OTS(25\%)-HZSM-5, (d) OTS(30\%)-HZSM-5, (e) OTS(35\%)-HZSM-5, and (f) OTS(40\%)-HZSM-5. by Han et $a l .{ }^{27}$ While preparing the catalyst, water was added to partly occupy the surface of the zeolite. The amphiphilic HZSM5 was obtained by adjusting the ratio of water and OTS. Since the number of $-\mathrm{O}-\left(\mathrm{Si}-\mathrm{CH}_{2}-\right)_{2}-\left(\mathrm{CH}_{2}\right)_{8}-\mathrm{CH}_{3}$ groups was different, the CA was changed with the different amount of OTS.

3.1.3 $\mathbf{N}_{2}$-adsorption/desorption analysis. The $\mathrm{N}_{2}$ adsorption isotherms of the HZSM-5 and OTS-HZSM-5 zeolites and the calculated pore structure parameters are shown in Fig. 4 and Table 1, respectively. The isotherms of the HZSM-5 and OTSHZSM-5 zeolites exhibited identical Langmuir type I isotherms. ${ }^{31,32}$ The OTS adsorption increased the resistance of the zeolite surface to $\mathrm{N}_{2}$ molecules, which made the hysteresis loop of OTS-HZSM-5 wider. The reason possibly was that the regularity of HZSM-5 was destroyed. Previous studies have reported that grafting organosilanes onto zeolites could reduce the surface area and pore volume as the pores were occupied by the organosilanes. ${ }^{33}$ After modifying with OTS, the BET surface area and the pore volume were reduced (the BET surface area changed from 311 to $144 \mathrm{~m}^{2} \mathrm{~g}^{-1}$, and the pore volume changed from 0.18 to $0.09 \mathrm{~cm}^{3} \mathrm{~g}^{-1}$ ). Since the kinetic diameter of OTS was larger than the ZSM-5 pore diameter (the intrinsic pore of ZSM-5 was $0.53 \mathrm{~nm} \times 0.56 \mathrm{~nm}),{ }^{27,34}$ OTS could not enter the pores, which the $\mathrm{C} 8$ hydrophobic chain was deposited on the outer surface of zeolite. The C8 hydrophobic chain deposited on the outer surface could improve the hydrophobicity of the zeolite. The silanol groups of the zeolite reacted with OTS to form "Si-O-Si" bonds, which could lead to the decline of the specific surface area and pore volume.

3.1.4 TPD and pyridine-IR analysis. The cyclohexene hydration reaction is an acid-catalyzed reaction; the acid sites of the porous structure played an important role in the catalytic performance..$^{35-37}$ The density of the Brønsted and Lewis acid sites in the zeolites depend on the $\mathrm{Si} / \mathrm{Al}$ ratio, the framework configuration, and the type of the cation used for charge compensation. $^{38}$ Moreover, it could be considered that the functionalization occurred preferentially on the external surface of the zeolite and caused a significant loss in the acid density.

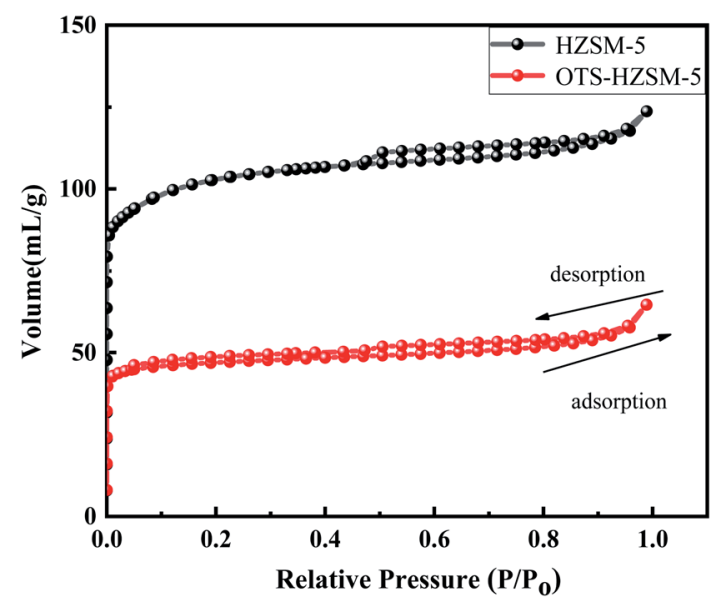

Fig. 4 Adsorption isotherms for nitrogen at $77 \mathrm{~K}$ on the HZSM-5 zeolite and OTS-HZSM-5 zeolite (Si/Al = 25). 
Table 1 Calculated composition of the HZSM-5 zeolite and OTSHZSM-5 zeolite $(\mathrm{Si} / \mathrm{Al}=25)$

\begin{tabular}{lllll}
\hline & & \multicolumn{3}{l}{ Pore volume $\left(\mathrm{cm}^{3} \mathrm{~g}^{-1}\right)$} \\
\cline { 3 - 5 } Sample & $\left(\mathrm{m}^{2} \mathrm{~g}^{-1}\right)$ & $V_{\text {total }}$ & $V_{\text {micro }}$ & $V_{\text {meso }}$ \\
\hline HZSM-5 & 311 & 0.18 & 0.12 & 0.06 \\
0TS-HZSM-5 & 144 & 0.09 & 0.07 & 0.02 \\
\hline
\end{tabular}

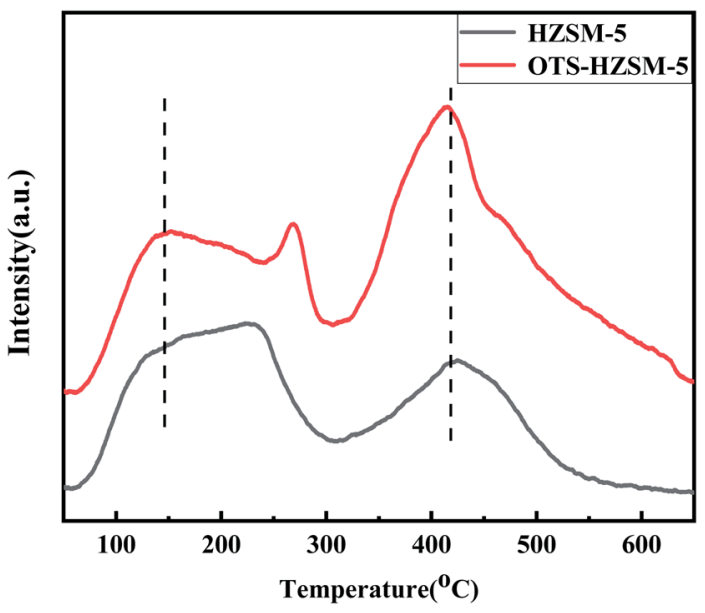

Fig. 5 TPD profile of the HZSM-5 zeolite and OTS-HZSM- 5 zeolite (Si/ $\mathrm{Al}=25)$.

To answer this question, the HZSM-5 and OTS-HZSM-5 zeolites were characterized via TPD and pyridine-IR.

In Fig. 5, $\mathrm{NH}_{3}$-TPD exhibited characteristic desorption peaks at $155{ }^{\circ} \mathrm{C}$ and $420{ }^{\circ} \mathrm{C}$; the desorption peak at a lower temperature corresponded to the weak acid center and the desorption peak at a higher temperature corresponded to the strong acid center. The OTS-HZSM-5 zeolite had a clear protruding profile, which corresponded to the thermal decomposition of OTS at approximately $250{ }^{\circ} \mathrm{C}$. It could be observed that the Brønsted

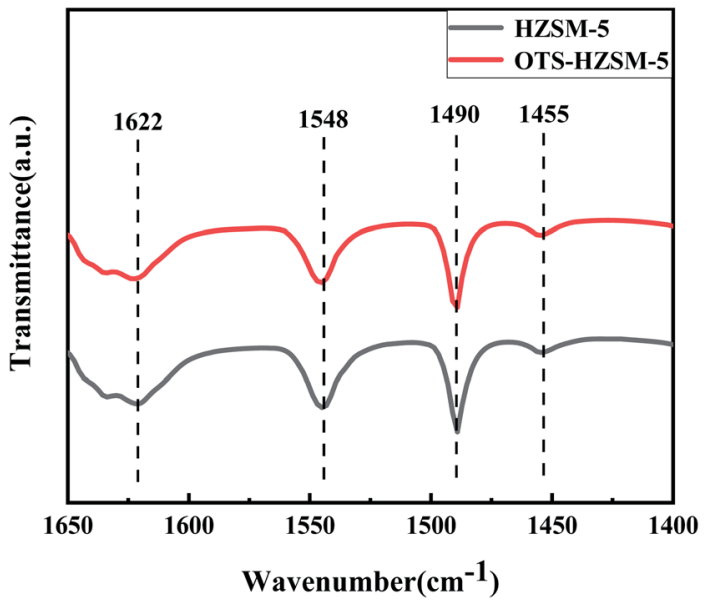

Fig. 6 Pyridine-IR spectra of the HZSM-5 zeolite and OTS-HZSM-5 zeolite at $200{ }^{\circ} \mathrm{C}$ and atmospheric pressure.
Table 2 Calculated acid of the HZSM-5 zeolite and OTS-HZSM-5 zeolite $(\mathrm{Si} / \mathrm{Al}=25)$

\begin{tabular}{|c|c|c|c|c|c|}
\hline \multirow[b]{2}{*}{ Sample } & \multicolumn{2}{|l|}{ Acid area } & \multicolumn{2}{|c|}{$\begin{array}{l}\text { Acid amount } \\
\left(\mathrm{mmol} \mathrm{g}^{-1}\right)\end{array}$} & \multirow{2}{*}{$\begin{array}{l}\text { Total acid } \\
\left(\mathrm{mmol} \mathrm{g}^{-1}\right)\end{array}$} \\
\hline & Brønsted & Lewis & Brønsted & Lewis & \\
\hline HZSM-5 & 8.69 & 1.64 & 352 & 88 & 441 \\
\hline 0TS-HZSM-5 & 8.01 & 1.38 & 319 & 62 & 382 \\
\hline
\end{tabular}

Table 3 Calculated composition of the HZSM-5 zeolite and OTS$\mathrm{HZSM}-5$ zeolite $(\mathrm{Si} / \mathrm{Al}=25)$

\begin{tabular}{llll}
\hline & \multicolumn{3}{c}{ Concentration of various elements $(\%)$} \\
\cline { 2 - 4 } Samples & $\mathrm{C}$ & $\mathrm{O}$ & $\mathrm{Si}$ \\
\hline HZSM-5 & 43.86 & 38.24 & 17.9 \\
OTS(25\%)-HZSM-5 & 45.96 & 36.91 & 17.13
\end{tabular}

and Lewis acid sites remained unchanged even after the modification.

In Fig. 6, the pyridine exhibited characteristic absorption bands at $1548 \mathrm{~cm}^{-1}, 1455 \mathrm{~cm}^{-1}, 1622 \mathrm{~cm}^{-1}$ and $1490 \mathrm{~cm}^{-1}$ after chemisorption on the acidic zeolites. These characteristic peaks corresponded to the Brønsted acid sites, the Lewis acid sites, and the central fractions of $\mathrm{B}$ and $\mathrm{L}$ acids. As shown in the spectra and Table 2, the peak intensity of OTS-HZSM-5 became weaker and the total acid content also decreased, which indicated that the Brønsted and Lewis acids slightly decreased after the OTS functionalization. It could be due to the fact that the $\mathrm{C} 8$ hydrophobic chain formed "Si-O-Si" bonds on the catalyst surface to cover part of the acidic sites.

3.1.5 XPS analysis. XPS is an important surface chemical analysis technique that could be utilized to analyze the surface property of a material. ${ }^{27}$ It could be observed from Table 3, and the effective coverage with organosilanes resulted in a strong increase in the carbon signal and a decrease in both the silicon

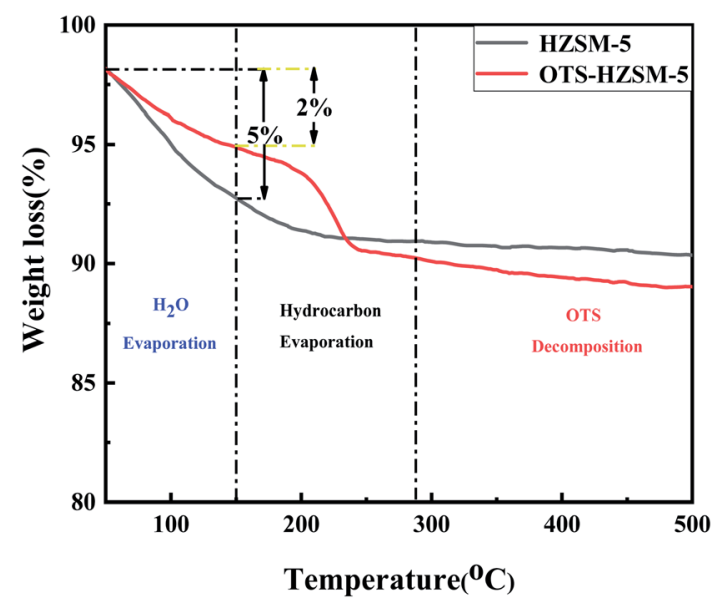

Fig. 7 Thermogravimetric analysis (TGA) of the HZSM-5 zeolite and OTS-HZSM-5 zeolite (Si/Al = 25); left axis: weight (\%). 


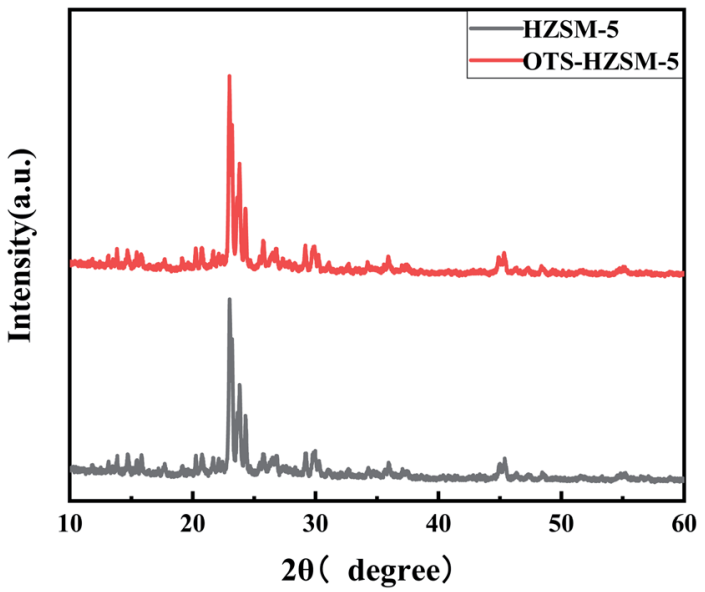

Fig. 8 XRD pattern of the HZSM-5 zeolite and OTS-HZSM-5 zeolite $(\mathrm{Si} / \mathrm{Al}=25)$
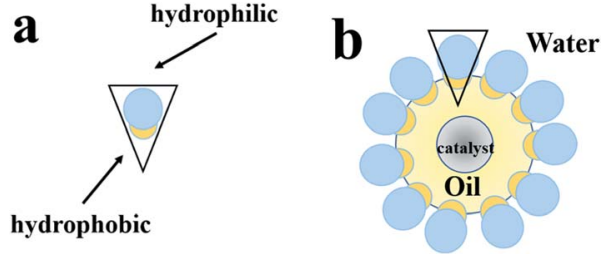

Fig. 9 Droplet composition of the OTS-HZSM-5 zeolite ( $a$ and b).

and oxygen signal intensities. ${ }^{39}$ The XPS studies suggested that the OTS could form covalent bonds in the HZSM-5 zeolite surface, forming an organic coating layer. This result is in agreement with the information obtained from FTIR spectroscopy.
3.1.6 TGA analysis. TGA was used to assess the stability of OTS-HZSM-5 and quantify the amount of OTS grafted onto HZSM-5. As shown in Fig. 7, the weight loss of the zeolite occurred in three steps: (1) $150{ }^{\circ} \mathrm{C}$, (2) $150-280{ }^{\circ} \mathrm{C}$, and (3) $280-$ $500{ }^{\circ} \mathrm{C}$. The weight loss was the hydrolysis absorption and evaporation of residual organic solvents in the first stage. OTSHZSM-5 (about 2\%) was lower than HZSM-5 (about 5\%), which was attributed to the fact that the HZSM-5 had more silanol groups. ${ }^{\mathbf{4 0 - 4 2}}$ This result suggested that the OTS-HZSM-5 zeolite was more hydrophobic than the HZSM-5 zeolite. Compared with HZSM-5, the weight loss of OTS-HZSM-5 had a larger decline in the second and third stages, ad this was attributed to the slow decomposition of OTS at a higher temperature. These changes indicated that OTS grafted onto the HZSM-5 zeolite had better hydrophobicity.

3.1.7 XRD analysis. The XRD pattern of OTS-HZSM-5 confirmed its high crystallinity, as shown in Fig. 8. These results suggested that HZSM-5 and OTS-HZSM-5 had the same crystallinity, indicating that the functionalization of the silanol groups could not change the topology structure of the zeolite crystals. $^{43}$

\subsection{Emulsion formation by modified catalysts in two-phase systems}

Since the surface of the zeolite contained numerous free silanol groups, HZSM-5 was hydrophilic. It could not be suspended in the lower polar cyclohexene phase, and the modified catalyst could be suspended in the middle of the oil-water phase interface. Thus, the catalyst suspension behaviors were investigated by placing HZSM-5 and OTS-HZSM-5 in cyclohexene hydration systems.

Fig. 10A illustrates the suspension of the untreated HZSM-5 zeolite. Due to the hydrophilic nature of zeolite, the HZSM-5 zeolite was at the bottom of the water phase in the watercyclohexene system. A single emulsion was formed by stirring,

(A) HZSM-5
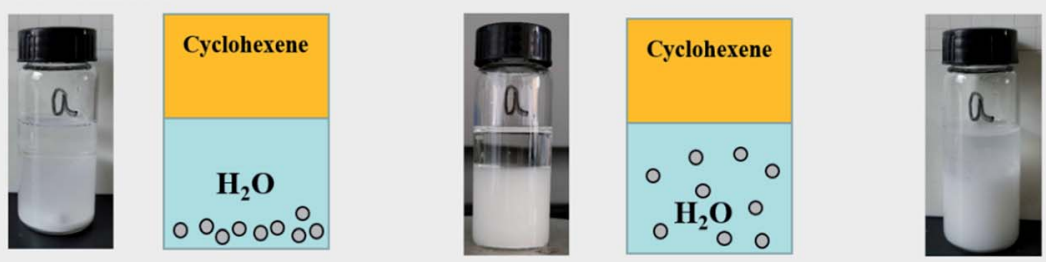

(B) OTS-HZSM-5
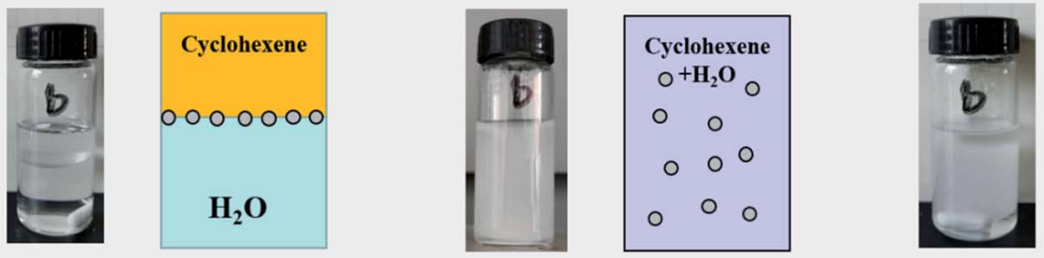

Fig. 10 Suspension behaviors of the HZSM-5 and OTS-HZSM-5 zeolites in the cyclohexene system (A and B). 


\section{(A) HZSM-5}
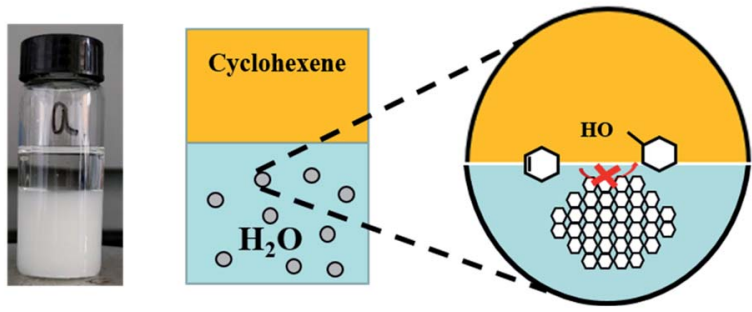

\section{(B) OTS-HZSM-5}
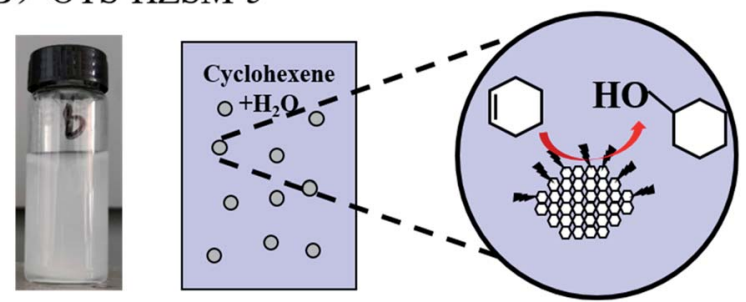

Fig. 11 The cyclohexene hydration of the HZSM-5 zeolite and OTSHZSM-5 zeolite (A and B).

and the contact between the catalyst and reactants was only a layer of the phase interface, which led to the low conversion in the cyclohexene hydration reaction (Fig. 11A). When the hydrophobic catalyst was added, OTS-HZSM-5 was suspended in a two-phase interface (Fig. 10B). For the OTS-HZSM-5 zeolite, a uniform Pickering emulsion was formed by stirring, which could increase the contact area of the two phases to improve the conversion (Fig. 11B).

The droplet of the three-layer structure was formed by stirring. By magnifying the droplet, it was found that the outermost layer of the zeolite was hydrophilic, the secondary outer layer was oleophilic and the inner layer was a solid catalyst (Fig. 9a and b). The reaction activity was restricted due to the mass transfer limitations, which restricted the contact between the reactants. OTS-HZSM- 5 could increase the contact between the two reactants, which formed a Pickering emulsion between the

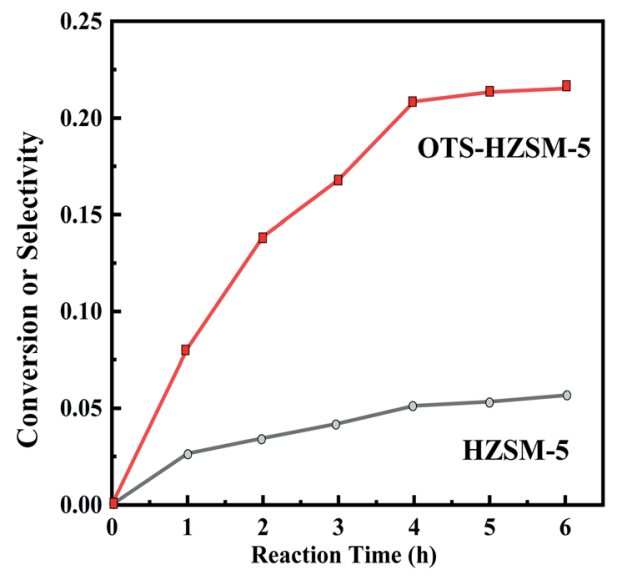

Fig. 12 Cyclohexene conversion of the HZSM-5 zeolite and OTSHZSM-5 zeolite in different reaction times.
Table 4 Cyclohexene conversion of the HZSM-5 zeolite and OTSHZSM- 5 zeolite in stir and static environments

\begin{tabular}{lcc}
\hline Type of catalyst & \multicolumn{2}{l}{$\begin{array}{l}\text { Stir conversion } \\
(\%)\end{array}$} \\
\hline HZSM-5 & 9.81 & $\begin{array}{l}\text { Static conversion } \\
(\%)\end{array}$ \\
OTS-HZSM-5 & 21.13 & 5.03 \\
\end{tabular}

two immiscible reactants (Fig. 10B). Therefore, the limitation of the interfacial mass transfer was minimized, which increased the cyclohexene conversion. These visual observations were consistent with the assumption that OTS was grafted onto HZSM-5 to add a hydrophobic layer on the HZSM-5 surface.

\subsection{Catalytic activity of OTS-HZSM-5 catalysts in cyclohexene hydration}

Fig. 12 compares the cyclohexene conversion between two zeolites at different reaction times. A clear difference is observed: OTS-HZSM- 5 had a good catalytic efficiency, and the cyclohexene conversion increased from 0 to $22.08 \%$ with reaction times. When the reaction time was increased from 0 to $6 \mathrm{~h}$, HZSM-5 was only $5.18 \%$.

The change of conversion was investigated under stirring and static environment in Table 4. Due to the fact that stirring could increase the contact area of the two reactants, the cyclohexene conversion significantly increased after stirring in the HZSM-5 zeolite. For the OTS-HZSM-5 zeolite, the cyclohexene conversion did not significantly improve. In the environment with a reaction temperature of $130{ }^{\circ} \mathrm{C}$, both water and cyclohexene reached boiling points. The boiling phenomenon caused the three phases to form a uniform Pickering emulsion, which greatly increased the contact area in the reaction system. Due to the need of the industrialization of catalytic distillation, the catalyst was fixed in the tower. Considering the abovementioned observations, we chose to evaluate the catalysts in the static state.

To further compare the stability of these catalysts in the reaction process, the following experiments were performed. The reactants (water, catalyst and cyclohexene) were added to a reaction kettle. After the reaction, the product was cooled in a water bath. The oil phase and water phase were separated via centrifugation, and then chromatographic analysis was performed with the quantitative addition of the internal standard ethanol. The separated solid catalyst was washed with ethyl alcohol and carbon tetrachloride, and dried in an oven overnight at $120{ }^{\circ} \mathrm{C}$. To maintain the hydrophobicity of the OTSHZSM-5 zeolite, no other high-temperature regeneration methods were used. Since a significant fraction of the catalyst was lost during the separation, washing, and drying processes, we repeated multiple sets of experiments and recovered them to evaluate the zeolite life experiment.

The OTS-HZSM-5 zeolites with different functionalization $(20 \%, 25 \%, 30 \%, 35 \%$, and $40 \%)$ were prepared by adjusting the ratio of water and (octyl)-trimethoxysilane (OTS). When the degree of silylation was $25 \%$, the conversion was as high as 


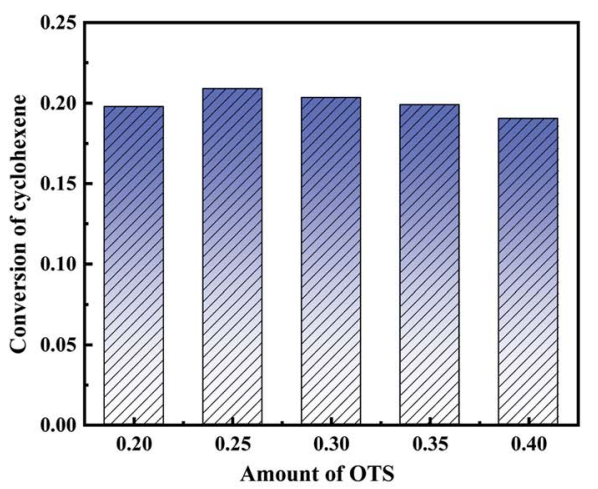

(a)

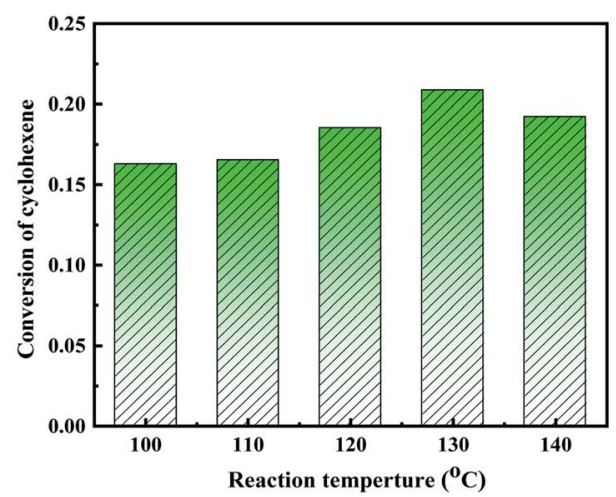

(c)

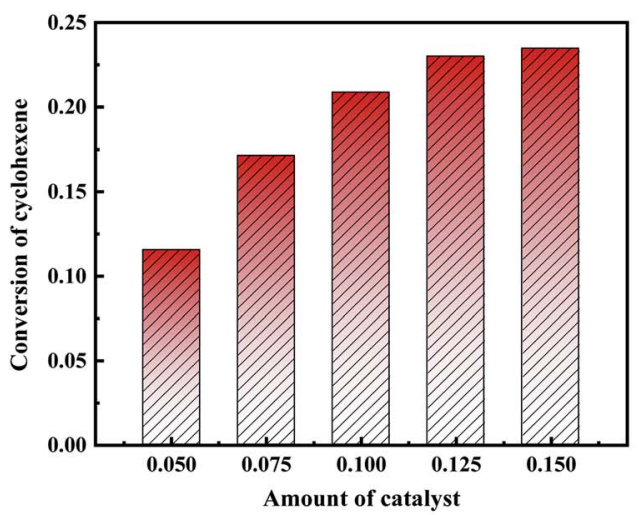

(e)

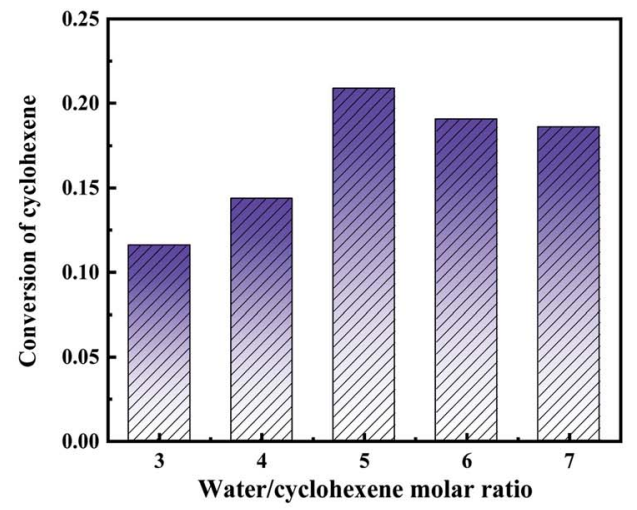

(b)

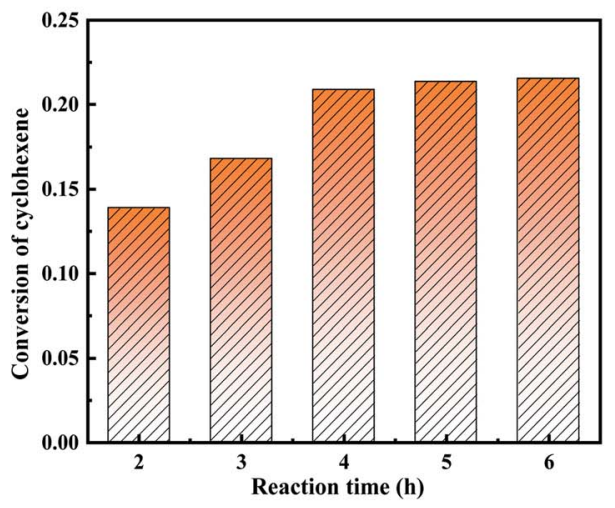

(d)

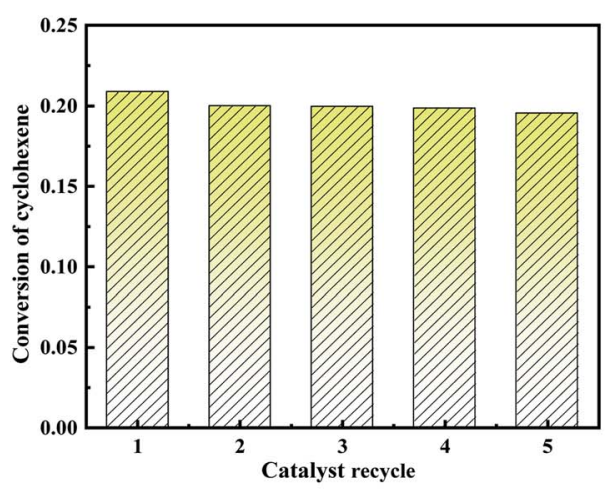

(f)

Fig. 13 Cyclohexene conversion of the HZSM-5 zeolite and OTS-HZSM-5 zeolite (Si/Al = 25). (a) The different functionalization (20\%, 25\%, 30\%, $35 \%$, and $40 \%$ ); (b) the different water-olefin molar ratio $(3,4,5,6$, and 7$)$; (c) the different reaction temperature $\left(110{ }^{\circ} \mathrm{C}, 120{ }^{\circ} \mathrm{C}, 130{ }^{\circ} \mathrm{C}, 140{ }^{\circ} \mathrm{C}\right.$, and $\left.150{ }^{\circ} \mathrm{C}\right)$; (d) the different reaction time $(2 \mathrm{~h}, 3 \mathrm{~h}, 4 \mathrm{~h}, 5 \mathrm{~h}$, and $6 \mathrm{~h})$; (e) the different amount of catalyst $(5 \%, 7.5 \%, 10 \%, 12.5 \%$, and $15 \%) ;(\mathrm{f})$ catalyst recycle $\left(1,2,3,4\right.$, and 5). Optimum reaction conditions: $\mathrm{OTS}(25 \%)$; water/cyclohexene molar ratios $=5$; reaction temperature $=130{ }^{\circ} \mathrm{C}$; reaction time $=4 \mathrm{~h}$; catalyst dosage $=10 \%$.

$20.87 \%$. When the degree of silylation further increased, the conversion decreased. Since the OTS could form "Si-O-Si" bonds with the surface of the HZSM-5 zeolite, the C8 hydrophobic chain could block a part of the pores and inactivate a part of the acidic sites. As the amount of the OTS reagent increased, the C8 hydrophobic chain could wrap the surface of the catalyst and reduce the specific surface area, thus decreasing cyclohexene conversion. So, the OTS(25\%)-HZSM-5 was chosen (Fig. 13a).

The cyclohexene hydration reaction was a three-phase coexistence reaction, different water-olefin molar ratios had different conversion, which proper molar ratios could promote the hydration reaction. The cyclohexene conversion was evaluated when the molar ratios of water-cyclohexene were 3, 4, 5, 6 
and 7 , respectively. It observed that the conversion first increased and then decreased. Excessive water could promote the reaction of carbocation with water molecules to produce protonated alcohols, which increased conversion. When water was added after the reaction reached equilibrium, the conversion could not increase significantly. So, the water-cyclohexene molar ratios were 5 (Fig. 13b).

The reaction temperature was investigated at $100{ }^{\circ} \mathrm{C}, 110{ }^{\circ} \mathrm{C}$, $120{ }^{\circ} \mathrm{C}, 130{ }^{\circ} \mathrm{C}, 140{ }^{\circ} \mathrm{C}$ and $150{ }^{\circ} \mathrm{C}$ in the cyclohexene hydration reaction. As the reaction temperature increased, the collision of molecules increased to improve the reactant contact. Furthermore, since cyclohexene hydration is a reversible exothermic reaction, when the reaction temperature was too high, the reaction proceeded in the reverse direction. So, the reaction temperature was considered as $140{ }^{\circ} \mathrm{C}$ (Fig. 13c).

The cyclohexene conversion showed an increasing trend and then stabilized with an increase in the reaction time. As the reaction time increased, the reaction slowly achieved equilibrium. So, the reaction time was considered as $4 \mathrm{~h}$ (Fig. 13d).

The amount the catalyst was investigated with mass percentages of $5 \%, 7.5 \%, 10 \%, 12.5 \%$ and $15 \%$ (relative to the quality of water). Increasing the amount of the catalyst could improve the dispersion in the aqueous and oil phases and reduce the interfacial mass transfer limitations. When the amount of the catalyst was too large, the cyclohexene conversion remained constant. This was because the reaction achieved the state of equilibrium. To ensure cost effectiveness, the catalyst mass fraction was considered as $10 \%$ (Fig. 13e).

In this group of experiments, the stability of the catalyst was studied by recycling the catalyst. Simultaneously, the OTSHZSM-5 zeolite retained a large fraction of activity ( 90\%) after regeneration. It was found that the color of the catalyst gradually changed from initial white to dark yellow as the number of the catalyst cycles increased. As the catalyst was used repeatedly, the polar products were adsorbed on the catalyst pores, which blocked the catalyst pores and gradually reduced the catalyst activity (Fig. 13f).

\section{Conclusion}

The cyclohexene hydration reaction was an acid-catalyzed reaction, and the functionalization of the silanol groups could improve hydrophobicity, which could not cause a significant loss in the acid density. The OTS-HZSM-5 zeolite was synthesized, which was confirmed by FT-IR, CA and BET characterizations. The catalytic performance was conducted in the cyclohexene hydration, and the results showed that the OTSHZSM-5 zeolite exhibited better stability and conversion (20.87\%). Compared with the unmodified HZSM-5, which was due to the OTS-HZSM-5 could form an uniform Pickering emulsion to increase the two phases contact area. Hydrophobic zeolites were promising for other acid-catalyzed reactions to enhance industrial applications.

\section{Conflicts of interest}

There are no conflicts to declare.

\section{Acknowledgements}

The authors acknowledge the Focus on Research and Development Plan in Yantai city (2018XSCC038), and the Qingchuang Science and Technology Plan Innovation Team of Shandong province (2020KJC002).

\section{References}

1 M. Robert and P. Martyn, A cleaner way to nylon?, Nature, 2005, 437, 1243-1244.

2 I. Hiroshi, Liquid-phase hydration process of cyclohexene with zeolites, Catal. Surv. Asia, 1997, 1, 241-246.

3 M. T. Musser, Cyclohexanol and Cyclohexanone, Ullmann's Encycl. Ind. Chem., 2011, 203, 1889-1896.

4 V. Majid and M. H. Mohammad, Efficient fatty acid esterification using silica supported Brønsted acidic ionic liquid catalyst: experimental study and DFT modeling, Chem. Eng. J., 2014, 221, 254-257.

5 J. B. Wen, K. Y. You, X. L. Liu, J. Jian, F. F. Zhao, P. L. Liu, Q. H. Ai and H. Luo, Highly selective one-step catalytic amination of cyclohexene to cyclohexylamine over HZSM-5, Catal. Commun., 2019, 127, 64-68.

6 R. Kumar, S. Shah, P. P. Das, G. G. Bhagavanbhai, A. A. Fatesh and B. Chowdhury, An overview of caprolactam synthesis, Catal. Rev.: Sci. Eng., 2019, 161, 1520-1523.

7 A. Li, K. Shen, J. Chen, Z. Li and Y. Li, Highly selective hydrogenation of phenol to cyclohexanol over MOF-derived non-noble Co-Ni@NC catalysts, Chem. Eng. Sci., 2017, 166, 66-76.

8 R. M. Ibberson, S. Parsons, D. R. Allan and A. M. T. Bell, Polymorphism in cyclohexanol, Acta Crystallogr., Sect. B: Struct. Sci., Cryst. Eng. Mater., 2008, 64, 573-582.

9 Y. Tang, B. Li, N. Zhang, S. Wang, Y. Wen, P. Jin and X. Wang, Growth of ZSM-5 zeolite microparticles from crystal seeds for catalytic hydration of cyclohexene, CrystEngComm, 2012, 14, 3854-3857.

10 X. Shan, Z. Cheng and Y. Li, Solvent Effects on Hydration of Cyclohexene over H-ZSM-5 Catalyst, J. Chem. Eng. Data, 2011, 56, 4310-4316.

11 B. Edoardo, P. Luca, R. Lucio, T. Claudio and V. Andrea, Trifluoroacetic acid promoted hydration of styrene catalyzed by sulfonic resins: comparison of the reactivity of styrene, n-hexene and cyclohexene, Appl. Catal., A, 2019, 570, 130-138.

12 P. Yuan, Y. Liu, F. Bai, L. Xu, Z. Cheng and W. Yuan, Hydration of cyclohexene in sub-critical water over WOx$\mathrm{ZrO}_{2}$ catalysts, Catal. Commun., 2011, 12, 753-756.

13 J. Li, L. Yang, F. Li, W. Xue and Y. Wang, Hydration of cyclohexene to cyclohexanol over SO3H-functionalized imidazole ionic liquids, React. Kinet., Mech. Catal., 2015, 114, 173-183.

14 H. Spod, L. Martin and C. Peter, Hydratisierung von Cyclohexen zu Cyclohexanol unter Verwendung von HZSM5 als Katalysator, Chem. Ing. Tech., 2017, 89, 750-756. 
15 H. Nur, S. Ikeda and B. Ohtani, Phase-boundary catalysts for acid-catalyzed reactions: the role of bimodal amphiphilic structure and location of active sites, J. Braz. Chem. Soc., 2004, 15, 719-724.

$16 \mathrm{~S} . \mathrm{D} . \mathrm{Xu}, \mathrm{H}$. D. Sheng, T. Ye, D. Hu and S. F. Liao, Hydrophobic aluminosilicate zeolites as highly efficient catalysts for the dehydration of alcohols, Catal. Commun., 2016, 78, 75-79.

17 P. A. Zapata, J. Faria, M. P. Ruiz, R. E. Jentoft and D. E. Resasco, Hydrophobic Zeolites for Biofuel Upgrading Reactions at the Liquid-Liquid Interface in Water/Oil Emulsions, Am. Chem. Soc., 2012, 134, 8570-8578.

18 C. Wang, S. Z. Leng, H. D. Guo, L. Y. Cao and J. F. Huang, Acid and alkali treatments for regulation of hydrophilicity/ hydrophobicity of natural zeolite, Appl. Surf. Sci., 2019, 478, 319-326.

19 D. Li, Z. Wu, D. Zhou, Y. Xia, X. Lu, H. He and Q. Xia, Onestep synthesis of hybrid zeolite with exceptional hydrophobicity to accelerate the interfacial reaction at low temperature, Microporous Mesoporous Mater., 2019, 280, 195-202.

20 A. Corma, P. Esteve and A. Martínez, Solvent Effects during the Oxidation of Olefins and Alcohols with Hydrogen Peroxide on Ti-Beta Catalyst: The Influence of the Hydrophilicity-Hydrophobicity of the Zeolite, Catal. Commun., 1996, 161, 11-19.

21 M. G. Ahunbay, Monte Carlo Simulation of Water Adsorption in Hydrophobic MFI Zeolites with Hydrophilic Sites, Langmuir, 2011, 27, 4986-4993.

22 J. Gallagher, Hydrophobic help, Nat. Energy, 2018, 9, 4378.

23 T. Merle, J. Pic, M. H. Manero and H. Debellefontaine, Comparison of Activated Carbon and Hydrophobic Zeolite Efficiencies in 2,4-Dichlorophenol Advanced Ozonation, Ozone: Sci. Eng., 2010, 32, 391-398.

24 S. Melki, F. Biguenet and D. Dupuis, Hydrophobic properties of textile materials: robustness of hydrophobicity, J. Text. Inst., 2019, 110, 1221-1228.

25 S. Crossley, J. Faria, F. Shen and D. E. Resasco, Solid Nanoparticles that Catalyze Biofuel Upgrade Reactions at the Water/Oil Interface, J. Text. Inst., 2010, 327, 68-72.

26 M. Pera-Titus, L. Leclercq, L. Clacens, F. D. Campo and V. Nardello-Rataj, Pickering interfacial catalysis for biphasic systems: from emulsion design to green reactions, Angew. Chem., Int. Ed., 2014, 53, 2-18.

27 X. L. Han, L. Wang, J. D. Li, X. Zhan, J. Chen and J. C. Yang, Tuning the hydrophobicity of ZSM-5 zeolites by surface silanization using alkyltrichlorosilane, Appl. Surf. Sci., 2011, 257, 9525-9531.

28 S. A. Kulkarni, S. A. Mirji, A. B. Mandale and K. P. Vijayamohanan, Thermal stability of self-assembled octadecyltrichlorosilane monolayers on planar and curved silica surfaces, Thin Solid Films, 2006, 496, 420-425.

29 T. I. Korányi, F. Moreau, V. V. Rozanov and E. A. Rozanova, Identification of $\mathrm{SH}$ groups in zeolite-supported HDS catalysts by FTIR spectroscopy, J. Mol. Struct., 1997, 410, 103-110.
30 Q. Zhengxing, L. Louwanda, T. Lubomira, G. Jean-Pierre, V. Aurélie, F. Christian and V. Valentin, Comparative Study of Nano-ZSM-5 Catalysts Synthesized in $\mathrm{OH}^{-}$and $\mathrm{F}^{-}$ Media, Adv. Funct. Mater., 2014, 24, 257-264.

31 L. Tosheva, V. Valtchev and J. Sterte, Silicalite-1 containing microspheres prepared using shape-directing macrotemplates, Microporous Mesoporous Mater., 2000, 36, 621629.

32 N. Viswanadham, R. Kamble, M. Singh, M. Kumar and D. G. Murali, Catalytic properties of nano-sized ZSM-5 aggregates, Catal. Today, 2009, 141, 182-186.

33 P. A. Zapata, Y. Huang, M. A. Gonzalez-Borja and D. E. Resasco, Silylated hydrophobic zeolites with enhanced tolerance to hot liquid water, J. Catal., 2013, 308, 82-97.

34 C. Chmelik, A. Varma, L. Heinke, D. B. Shah, J. Karger, F. Kremer, U. Wilczok and W. Schmidt, Effect of Surface Modification on Uptake Rates of Isobutane in MFI Crystals: An Infrared Microscopy Study, Chem. Mater., 2007, 19, 6012-6019.

35 E. Bianchini, L. Pietrobon, L. Ronchin, C. Tortato and A. Vavasori, Trifluoroacetic acid promoted hydration of styrene catalyzed by sulfonic resins: comparison of the reactivity of styrene, n-hexene and cyclohexene, Appl. Catal., A, 2019, 570, 130-138.

36 G. Liu, M. Chen, X. Jin, C. Song, F. He and Q. Huang, Combination of $\mathrm{H}_{3} \mathrm{PW}_{12} \mathrm{O}_{40}-\mathrm{TiO}_{2}$ catalysts for photothermal oxidation of cyclohexene to adipic acid by $30 \%$ $\mathrm{H}_{2} \mathrm{O}_{2}$, J. Environ. Chem. Eng., 2021, 105422.

37 Y. Choi, H. Park, Y. Yun and J. Yi, Effects of catalyst pore structure and acid properties on the dehydration of glycerol, ChemSusChem, 2015, 8, 974-979.

38 X. G. Wang, Y. Zhao, Y. W. Tang and T. Lin, Superhydrophobic cotton fabric fabricated by electrostatic assembly of silica nanoparticles and its remarkable buoyancy, Appl. Surf. Sci., 2010, 256, 6736-6742.

39 Z. W. Li and Y. F. Zhu, Surface-modification of $\mathrm{SiO}_{2}$ nanoparticles with oleic acid, Appl. Surf. Sci., 2003, 211, 315-320.

40 I. S. Chuang and G. E. Maciel, Probing Hydrogen Bonding and the Local Environment of Silanols on Silica Surfaces via Nuclear Spin Cross Polarization Dynamics, Am. Chem. Soc., 1996, 118, 401-406.

41 S. A. Mirji, S. B. Halligudi, D. P. Sawant, N. E. Jacob, K. R. Patil, A. B. Gaikwad and S. D. Pradhan, Adsorption of octadecyltrichlorosilane on mesoporous SBA-15, Appl. Surf. Sci., 2006, 252, 4097-4103.

42 V. B. Kazansky, A. I. Serykh, V. Semmer-Herledan and J. Frasissard, Intensities of OH IR stretching bands as a measure of the intrinsic acidity of bridging hydroxyl groups in zeolites, Phys. Chem., 2003, 5, 966-969.

43 V. Felice and A. C. Tavares, Faujasite zeolites as solid electrolyte for low temperature fuel cells, Solid State Ionics, 2011, 194, 53-61. 